\title{
Advances in Cellular Characterization of the Sirtuin Isoform, SIRT7
}

\author{
Di Wu ${ }^{1,2+}$, Yinglu $\mathrm{Li}^{3 \dagger}$, Kathy S. Zhu ${ }^{4}$, Haiying Wang ${ }^{1,5 *}$ and Wei-Guo Zhu ${ }^{3 *}$ \\ ${ }^{1}$ Key Laboratory of Carcinogenesis and Translational Research (Ministry of Education), Beijing Key Laboratory of Protein \\ Posttranslational Modifications and Cell Function, Peking University Health Science Center, School of Basic Medical \\ Sciences, Beijing, China, ${ }^{2}$ School of Basic Medical Sciences, Institute of Systems Biomedicine, Peking University Health \\ Science Center, Beijing, China, ${ }^{3}$ Department of Biochemistry and Molecular Biology, Shenzhen University Health Science \\ Center, Shenzhen, China, ${ }^{4}$ Peking University Health Science Center, School of Public Health, Beijing, China, ${ }^{5}$ Department of \\ Biochemistry and Molecular Biology, Peking University Health Science Center, School of Basic Medical Sciences, Beijing, \\ China
}

SIRT7 is one of seven mammalian sirtuins that functions as an $\mathrm{NAD}^{+}$-dependent histone/protein deacetylase. SIRT7 is the least well-known member of the sirtuin family,

\section{OPEN ACCESS}

Edited by:

Yang Yang,

Northwest University, China

Reviewed by:

Adam John Watkins, University of Nottingham

United Kingdom

Carmen Jeronimo,

IPO Porto, Portugal

Tammy A. Morrish, Independent Researcher

United States

*Correspondence:

Wei-Guo Zhu

zhuweiguo@bjmu.edu.cn;

zhuweiguo@szu.edu.cn

Haiying Wang

wendy@bjmu.edu.cn

tThese authors have contributed equally to this work

Specialty section: This article was submitted to

Cellular Endocrinology

a section of the journal

Frontiers in Endocrinology

Received: 23 August 2018 Accepted: 17 October 2018 Published: 19 November 2018

Citation:

Wu D, Li Y, Zhu KS, Wang H and Zhu W-G (2018) Advances in Cellular

Characterization of the Sirtuin Isoform, SIRT7. Front. Endocrinol. 9:652 doi: 10.3389/fendo.2018.00652 but recent efforts have identified its involvement in various cellular processes, such as ribosome biogenesis, gene expression, cellular metabolism and cancer. Here we provide an update on the functions and mechanisms of SIRT7 in cellular regulation and disease.

Keywords: SIRT7, rDNA transcription, ribosome biogenesis, cellular stress, metabolism, aging, genome stability, cancer

\section{INTRODUCTION}

In the year 2000, researchers identified that silent information regulator 2 (Sir2)-a nicotinamide adenine dinucleotide (NAD)-dependent histone deacetylase-extends the lifespan of yeast $(1,2)$. Since then, the Sir2 homologs in mammals, known as sirtuins, have received increasing attention. There are seven sirtuin homologs in mammals (SIRT1 to SIRT7) that share a conserved NAD ${ }^{+}$ binding domain, but their cellular localization, activities and functions differ (Figure 1). SIRT1, SIRT6, and SIRT7 predominantly localize to the nucleus (SIRT7 primarily resides in nucleolus), SIRT2 localizes to the cytoplasm and SIRT3, SIRT4, and SIRT5 localize to the mitochondria (3). Sirtuins mediate various cellular functions that regulate a wide range of physiological processes, including the cell cycle, proliferation, apoptosis, aging, genomic stability, stress resistance and metabolism (4). SIRT7 is the least well-studied of the sirtuins, but recent breakthroughs have shown that it is also involved in numerous cellular processes and its biological function is gradually becoming clear. In this review, we outline the current data regarding SIRT7 function and highlight the areas where SIRT7 may have a possible therapeutic role in disease.

\section{SIRT7 ENZYMATIC ACTIVITY}

SIRT7 encodes a 400 amino acid protein that functions as a class III histone deacetylase in Homo sapiens. (5) Compared to other nuclear-localized Sirtuins (SIRT1 and SIRT6), SIRT7 exhibits weak deacetylase activity (3). Within the SIRT7 catalytic domain, serine residue 111 (S111) and histidine residue 187 (H187) are responsible for the deacetylation activity $(6,7)$, but to date, only a few SIRT7 substrates have been identified. In 2012, Barber et al. (6) reported that SIRT7 is a highly selective histone $\mathrm{H} 3$ lysine 18 (H3K18) deacetylase, and that H3K18Ac deacetylation is associated with high-grade tumors and poor patient prognosis. Non-histone substrates have also been identified, including p53, PAF53, U3-55k, GABP $\beta 1$, NPM1, PGK1, CDK9, DDB1, FKBP51, FOXO3, SMAD4, and DDX21 (Table 1). We discuss the data for each of these substrates in turn below. 


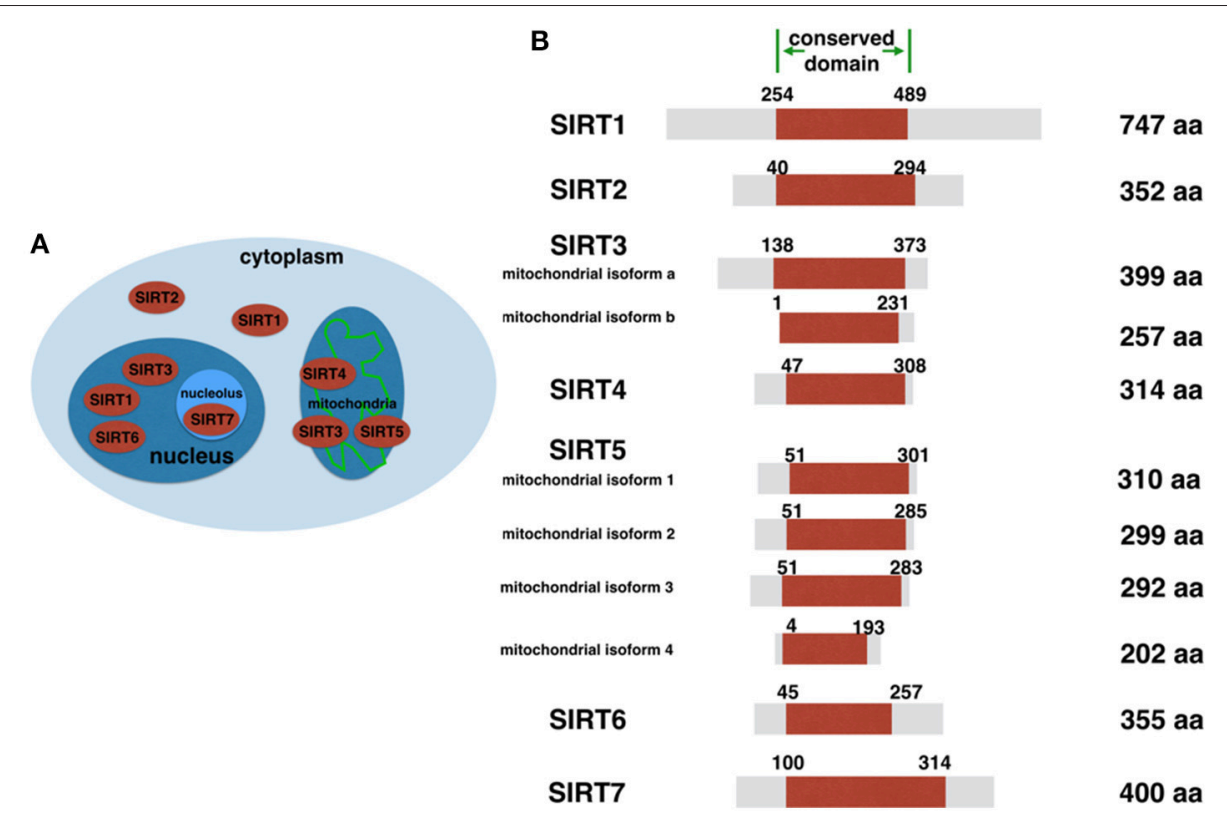

FIGURE 1 | Sirtuin family. (A) Sirtuins' intracellular location. (B) Human sirtuin proteins and their protein structures. The sequences are based on NCBI Protein database.

Vakhrusheva et al. found that p53 peptides were deacetylated in vitro by SIRT7 as efficiently as SIRT1, and mutant SIRT7 nearly abolished p53 decetylation (8). Conversely, Barber et al. did not detect SIRT7 deacetylase activity toward p53 both in vivo and in vitro, which questioned the ability of SIRT7 to deacetylate p53 (6). Kim et al. found an inverse correlation between p53 acetylation levels and SIRT7 expression in Hep3B cells (9). Finally, Nahalkova et al. identified an interaction between SIRT7 and p53, but did not assess SIRT7 deacetylase activity (10). p53 is debatable to be a SIRT7 substrate, for SIRT7 is found only effective toward $\mathrm{p} 53$ peptide, but not $\mathrm{p} 53$ protein both in vivo and in vitro. More studies are required, therefore, to resolve whether p53 is truly a SIRT7 substrate.

The wide variety of non-histone SIRT7 substrates suggests that SIRT7 participates in a diverse range of cellular processes. For example, SIRT7 can deacetylate nucleolar organizer polymeraseassociated factor 53 (PAF53) and U3-specific protein U3-55k to regulate the synthesis and ripening of precursor ribose RNA $(11,12)$. By bioinformatic analysis, Ryu et al. found that GAbinding protein $\beta 1(\mathrm{GABP} \beta 1)$ is another substrate of SIRT7 (13). Here, SIRT7 interacts with and deacetylates GABP $\beta 1$ to regulate mitochondrion function (13). Lee et al. reported that SIRT7 deacetylates nucleophosmin (NPM1), which is involved in regulating aging processes (14), while $\mathrm{Hu}$ et al. found that SIRT7 deacetylates phosphoglycerate kinase 1 (PGK1) and participates in glycolysis regulation (15). Yu et al. showed that SIRT7 specifically interacts with and deacetylates FK506binding protein 51 (FKBP51) at lysine residues 28 and 155 (K28 and K155), which enhances FKBP51-Akt-PHLPP complex formation, resulting in AKT inactivation and enhanced chemosensitivity in breast cancer cells (16). Blank et al. showed that SIRT7-dependent deacetylation of Cyclin-dependent kinase
9 (CDK9) activates its kinase activity; CDK9 subsequently phosphorylates the Pol II C-terminal domain (CTD) and facilitates transcription elongation (17). Li et al. observed that SIRT1/SIRT7 deacetylate FOXO3 in vitro and in vivo to prevent its phosphorylation and block apoptosis in response to lipopolysaccharide stimulation (18). Mo et al. found that SIRT7 is a major deacetylase of DNA damage-binding protein 1 (DDB1): DDB1 acetylation negatively regulates the DDB1-CUL4 interaction and CRL4 activity $(19,20)$. Tang et al. found that SIRT7 inhibits breast cancer lung metastasis by deacetylating and promoting SMAD4 degradation (21). Finally, Song et al. showed that deacetylation of the nucleolar DEAD-box helicase DDX21 by SIRT7 increases R-loop-unwinding activity and safeguards genome stability (22).

To widen the range of SIRT7 targets, our laboratory conducted the first proteomic screen of SIRT7 substrates. Using stable isotope labeling with amino acids in cell culture coupled with quantitative mass spectrometry, we produced a comprehensive list of candidates that are involved in a variety of functions, ranging from chromatin architecture homeostasis to gene silencing and metabolism. Some selected candidates, such as SIRT2, histone-lysine N-methyltransferase (EZH2), mitogen-activated protein kinase 2 (MAPK2) and glycogen synthase kinase-3 beta (GSK3 $\beta$ ) have been validated by in vitro co-immunoprecipitation and deacetylation experiments. By combining this approach with predictive tools, we have started to greatly expand the list of SIRT7 candidate substrates. Such studies have enhanced our understanding of the physiological role of SIRT7 and raised awareness as to the global impact of sirtuins in cell homeostasis (23).

SIRT7 exhibits other enzymatic activities in addition to $\mathrm{NAD}^{+}$-dependent deacetylation. In 2016, Li et al. found 
TABLE 1 | SIRT7 catalytic substrates.

\begin{tabular}{|c|c|c|}
\hline Substrate & Modification & Function \\
\hline H3K18 & Deacetylation & Tumorigenesis \\
\hline p53 & Deacetylation & Apoptosis \\
\hline $\begin{array}{l}\text { PAF53 and } \\
\text { U3-55k }\end{array}$ & Deacetylation & Synthesis and ripening of precursor ribose RNA \\
\hline GABP $\beta 1$ & Deacetylation & Mitochondrial function \\
\hline NPM1 & Deacetylation & Aging \\
\hline PGK1 & Deacetylation & Glycolysis \\
\hline FKBP51 & Deacetylation & $\begin{array}{l}\text { AKT inactivation and chemo-sensitivity in } \\
\text { breast cancer cells }\end{array}$ \\
\hline CDK9 & Deacetylation & Transcription elongation \\
\hline FOXO3 & Deacetylation & Blocks apoptosis in response to LPS \\
\hline DDB1 & Deacetylation & $\begin{array}{l}\text { Regulates DDB1-CUL4 interaction and CRL4 } \\
\text { activity }\end{array}$ \\
\hline SMAD4 & Deacetylation & Inhibits breast cancer lung metastasis \\
\hline DDX21 & Deacetylation & Genome stability \\
\hline H3K122 & Desuccinylation & Chromatin remodeling during DNA repair \\
\hline H3K36/K37 & $\begin{array}{l}\text { Deacetylation/ } \\
\text { debutyrylation }\end{array}$ & Unknown \\
\hline
\end{tabular}

that SIRT7 is an $\mathrm{NAD}^{+}$-dependent histone desuccinylase, and desuccinylates $\mathrm{H} 3 \mathrm{~K} 122$ to regulate chromatin remodeling during DNA repair (24). However, no non-histone substrates for SIRT7 desuccinylase have been identified. SIRT7 also interacts with some proteins without deacetylating them, such as mTOR, Myc, and TFIIIC2 (25); it is possible that SIRT7 regulates these proteins via its desuccinylase activity. Future investigations should investigate the spectrum of non-histone desuccinylation-mediated regulation by SIRT7. A recent report also showed that SIRT7 has robust deacetylase or debutyrylase activity on acetylated or butyrylated nucleosomes, mainly toward H3K36/K37 (26).

SIRT7 deacetylase activity is markedly enhanced by chromatin compositional DNA and RNA. SIRT7 also exhibits defattyacylase activity, which can be effectively activated by RNA $(27,28)$. Conversely, Myb-binding protein la (Mybbpla), a component of the chromatin remodeling complex B-WICH, inhibits SIRT7 deacetylation activity and increases H3K18 levels in cells (29), although the mechanistic details remain to be understood.

\section{SIRT7 EXPRESSION AND REGULATION}

SIRT7 is widely expressed in different organs and tissues of the human body: the highest expression is found in hyperplastic tissues, such as the spleen, liver and testis, and the lowest expression is found in the skeletal muscle, heart and brain (30). SIRT7 expression levels are associated with cellular proliferation, differentiation and the stress response, acting as a positive or negative regulator in different organs and tissues (31). Recent findings from the TCGA database suggest that SIRT7 expression is tightly correlated with the development of various types of cancer (32-34). The diverse roles of SIRT7 suggest it is tightly regulated by multiple mechanisms, as evidenced by SIRT1 $(35,36)$.

Previous reports have indicated that SIRT7 is regulated at the transcriptional, post-transcriptional and translational levels (Figure 2). At the transcriptional level, SIRT7 is regulated by upstream molecules, such as X-box binding protein 1 (XBP1), CCAAT-enhancer-binding protein $\alpha(\mathrm{C} / \mathrm{EBP} \alpha)$, and histone deacetylase 3 (HDAC3) $(37,38)$. At the post-transcriptional level, SIRT7 is negatively regulated by several microRNAs, such as hsamiR-125b, miR-125a-5p, miR-125b, miR-93, miR-3666, and miR$340(9,39-42)$. There are only few reports, however, describing how SIRT7 is regulated by post-translational modifications. Grob et al. found that SIRT7 is phosphorylated during mitosis by the cyclin-dependent kinase 1 (CDK1)-cyclin B pathway, although the phosphorylation sites and function have not been defined (43). Sun et al. found that SIRT7 is phosphorylated during cellular energy stress by 5 ' AMP-activated protein kinase (AMPK), which has a crucial role in determining SIRT7 subcellular distribution and degradation (44). The researchers also showed that SIRT7 is modified by polyubiquitination (44), which is consistent with our study that showed that SIRT7 is modified by Lys63-linked polyubiquitination (45). We also found that SIRT7 enzymatic activity is negatively regulated by ubiquitin-specificprocessing protease 7 (USP7)-mediated deubiquitination, which helps control gluconeogenesis by regulating G6PC expression (45). Post-translational modifications have a crucial role in regulating protein stability, activity, structure and function; thus, further studies into SIRT7 post-translational modifications are warranted.

\section{SIRT7 BIOLOGICAL FUNCTIONS Ribosome Biogenesis and Protein Translation}

Global proteomic studies have identified numerous SIRT7associated proteins, with most of them having important roles in transcription, ribosome biogenesis and translation (7, 14, 25). Subsequent functional studies have confirmed that SIRT7 is involved in multiple pathways regulating ribosome biogenesis and protein translation. First, SIRT7 can regulate rDNA transcription. Ford et al. found that SIRT7 forms part of the RNA Polymerase I (RNA Pol I) complex, and activation of the RNA Pol I transcription in vivo is dependent on its enzymatic activity (30). Specifically, the researchers showed that SIRT7 over-expression increased Pol I-mediated transcription, whereas SIRT7 knockdown or catalytic inhibition decreased Pol I transcription (30). Grob et al. also found that SIRT7 activates Pol I transcription by interacting with upstream binding factor (UBF), which is a component of the RNA polymerase complex (43). Tsai et al. extended the mechanisms of SIRT7mediated rDNA transcriptional regulation by showing that SIRT7 associates with the B-WICH complex, a chromatin remodeling complex involved in rDNA transcriptional regulation and chromatin structure changes. In addition, the researchers found that down-regulated SIRT7 reduced levels of RNA Pol I protein by decreasing the expression levels of RPA194, the 


\section{A Transcription}
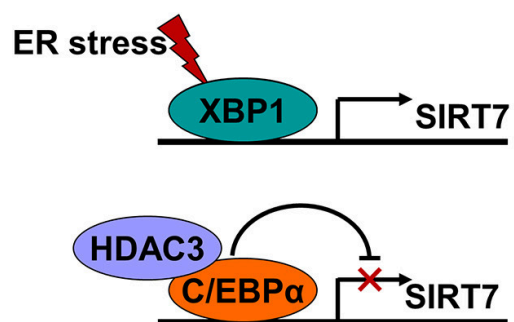

hsa-miR-125b/miR-125a-5p/miR125b/miR-93/miR-3666/miR-340

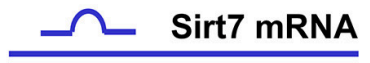

B Post-translational modifications
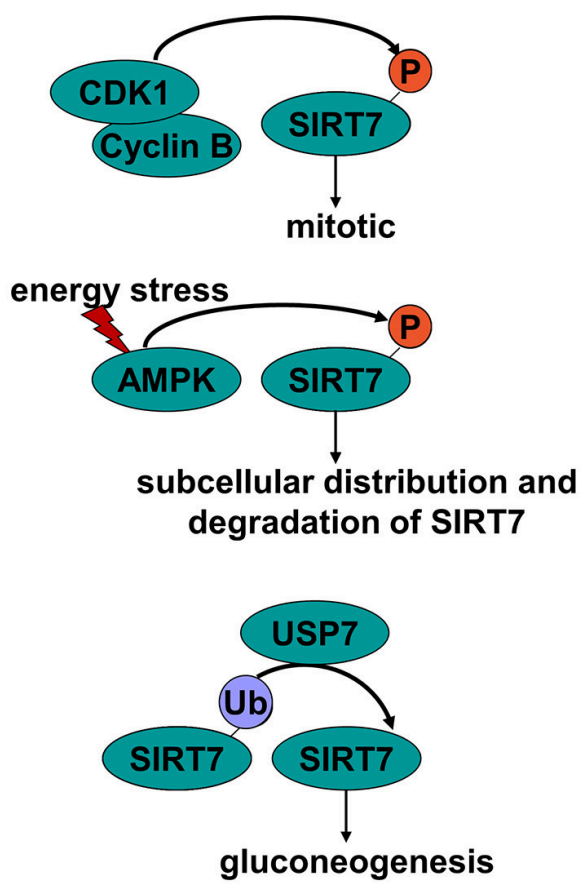

FIGURE 2 | Summary of regulation of SIRT7. (A) Various transcription factors regulate SIRT7 expression. The X-box binding protein 1 (XBP1) enhances SIRT7 expression, whereas Histone Deacetylase 3 (HDAC3) and CCAAT/enhancer-binding protein alpha (C/EBP alpha) repress SIRT7 expression. In addition, SIRT7 expression is also repressed by the microRNAs, such as hsa-miR-125b, miR-125a-5p, miR-125b, miR-93, miR-3666, and miR-340. (B) Some post-translational modifications affect SIRT7 activity. The cyclin-dependent kinase 1 (CDK1)-cyclin B pathway phosphorylates SIRT7 during mitosis. Under energy stress, SIRT7 can be phosphorylated by Adenosine $5^{\prime}$-monophosphate (AMP)-activated protein kinase (AMPK), thereby determining the subcellular distribution and degradation of SIRT7. Moreover, Ubiquitin-specific protease 7 (USP7) negatively regulates the enzymatic activity of SIRT7 through deubiquitination to control gluconeogenesis.

largest RNA polymerase I subunit (7). Others have shown that SIRT7 regulates Pol I transcription by deacetylating PAF53, another component of RNA polymerase I complex: PAF53 hypoacetylation correlates with increased Pol I occupancy on rDNA and transcriptional activation (12).

SIRT7 also regulates the conversion between rDNA transcriptional activation and inhibition during the cell cycle. Here, SIRT7 is phosphorylated by the CDK1-cyclin B pathway during mitosis, rendering SIRT7 inactive such that rDNA transcription is inhibited. Upon exiting mitosis, SIRT7 is dephosphorylated by a phosphatase sensitive to okadaic acid such that rDNA transcription can resume (43).

SIRT7 is also involved in pre-rRNA processing and rRNA maturation. SIRT7 can deacetylate U3-55k, a component of the U3 small nucleolar RNP (snRNP) complex, which increases the association between U3-55k and U3 snRNP. This association is essential for processing pre-rRNA. Under stress conditions however, SIRT7 is released from the nucleoli, resulting in U3-55k hyper-acetylation and reduced pre-rRNA processing (11).

A role for SIRT7 in protein synthesis has also been suggested, as supported by Tsai et al. who found that SIRT7 knockdown suppresses both RNA and protein synthesis (25). They showed that SIRT7 associates with mTOR and TFIIIC2 to modulate Pol III-dependent tRNA transcription, and co-localizes with Pol III target genes. Supporting these observations, SIRT7 knockdown decreased tRNA levels and amino acid incorporation rates; however, SIRT7 over-expression did not increase the rate of protein synthesis, indicating that the observed reduction in protein synthesis may be an indirect effect of SIRT7 knockdown. In addition, the reduced amino acid incorporation rates in SIRT7 knockdown cells were due to reduced abundance of tRNAs for different amino acids (25).

Shin et al. found that Myc depletion significantly reduces SIRT7 occupancy at the promoters of ribosomal proteins. SIRT7 is targeted to the promoters of ribosomal proteins by interacting with Myc and repressing Myc-dependent expression (38).

Finally, SIRT7 may have a role in Pol II activation, as data indicate that SIRT7 associates with Pol II and regulates transcription of snoRNAs and other Pol II genes (17). Here, SIRT7 promotes the release of the elongation factor $\mathrm{P}-\mathrm{TEFb}$ from the inactive 7SK snRNP complex and deacetylates CDK9, a component of the P-TEFb complex. CDK9 deacetylation promotes Pol II C-terminal domain (CTD) phosphorylation and transcription elongation (17).

\section{Regulator of Cellular Stress}

SIRT7 is resistant to various cellular stressors, such as endoplasmic reticulum (ER) stress, oxidative stress, mitochondrial protein folding stress, nutrition stress and 
genotoxic stress $(8,12,38,46)$. As such, it can be inferred that SIRT7 has an important role in regulating cell survival. The function of SIRT7 in regulating rRNA and protein synthesis also supports a role for SIRT7 in cellular stress, because both processes are reduced under stressed conditions.

\section{ER Stress}

The ER is an important intracellular organelle where protein synthesis, folding, modification and trafficking occur. The accumulation of unfolded proteins or depletion of calcium stores triggers the ER stress response (also known as the unfolded protein response, UPR), to restore protein homeostasis by increasing the expression of molecular chaperones, decreasing protein translation and degrading unfolded proteins (47).

SIRT7 can relieve ER stress in two ways. First, under stress conditions, XBP1 induces SIRT7 expression, which in turn reduces ER stress response protein expression, such as $\mathrm{CHOP}$, XBP1s, and GRP78 (38). Second, SIRT7 can interact with Myc to facilitate its recruitment to the promoters of ribosomal proteins, such as RPS20 and RPS14 to repress their gene expression (38).

\section{Mitochondrial Stress}

The mitochondrion is an important organelle for regulating cellular energy homeostasis, and is thus sensitive to many stresses. Cellular stress leads to an accumulation of unfolded mitochondrial proteins resulting in mitochondrial protein folding stress $\left(\mathrm{PFS}^{\mathrm{mt}}\right)$ and the unfolded protein response in mitochondria $\left(\mathrm{UPR}^{\mathrm{mt}}\right.$ ) (48). Mohrin et al. found that SIRT7 alleviates $\mathrm{PFS}^{\mathrm{mt}}$ by repressing NRF1 activity and reducing the expression of the mitochondrial translation machinery (46). NRF1 is a master regulator of mitochondria, and by interacting with SIRT7, targets it to the promoters of mitochondrial ribosomal proteins ( $\mathrm{mRPs}$ ) and mitochondrial translation factors (mTFs) to repress their expression. This mechanism helps alleviate $\mathrm{PFS}^{\mathrm{mt}}$ and improve cellular survival under conditions of nutrient deprivation (46).

\section{Oxidative Stress}

Many diseases and disorders have been linked with a cellular oxidant-antioxidant imbalance. Data suggest that sirtuins are important in the homeostasis of cellular oxidation-reduction systems. Hypoxia-inducible factors HIF-1 and HIF-2 are essential transcription factors that mediate adaptation to hypoxia (49). Hubbi et al. found that SIRT7 interacts with HIF-1 $\alpha$ and HIF- $2 \alpha$ proteins and negatively regulates their expression (50). Over-expression of SIRT7 reduced the levels of HIF proteins as well as their transcriptional targets, independent of SIRT7 deacetylase activity and hydroxylation-mediated ubiquitinylation and the proteasomal and lysosomal-mediated degradation pathways. Vakhrusheva et al. found that SIRT7-deficient primary cardiomyocytes exhibit a drastic increase in basal apoptosis compared to wild-type primary cardiomyocytes upon exposure to hydrogen peroxide, suggesting a critical role for SIRT7 in regulating the oxidative stress response and cell death in the heart (8). The researchers speculated that this susceptibility of SIRT7 mutant cells to apoptosis may be due to hyperactive p53, as SIRT7 deacetylates p53 (8). Lewinska et al. also reported that vascular smooth muscle cells exposed to curcumin to induce oxidative damage, exhibited down-regulated SIRT7 and p53 stability (51). SIRT7 down-regulation also decreased Pol I mediated transcription, and the stabilized p53 activated its target protein p21, resulting in cell-cycle arrest. Thus, SIRT7 has a potential role in the resistance to different conditions of oxidative stress.

\section{Cardiac Injury}

SIRT7 has a role in cardiac homeostasis as illustrated by SIRT7 knockout mice that suffer from degenerative heart hypertrophy, as evidenced by cardiac cell fibrosis and inflammation, resulting in inflammatory cardiomyopathy (8). SIRT7 knockout mice also show increased blood lactate levels and decreased endurance to physical activity, due to oxygen insufficiency and decreased oxygen consumption by cardiac muscles stemming from mitochondrial respiratory dysfunction (13). A possible pathway by which SIRT7 maintains cardiac health may be through GABP. SIRT7 promotes GABP complex formation and activation by deacetylating GABP $\beta 1$ to enhance the expression of mitochondrial genes and promote mitochondrial respiration (13).

Araki et al. described another SIRT7 pathway that may regulate cardiac health (52). The researchers noted that SIRT7 expression increases at active wound healing sites upon acute cardiovascular injury and thus speculated that SIRT7 may be involved in tissue repair. Consistently, SIRT7 depletion led to reduced collagen production and insufficient angiogenic and inflammatory responses, resulting in impaired wound healing (52). TGF- $\beta$ is essential to wound healing as it regulates fibroblast chemotaxis, differentiation and the epithelialto-mesenchymal transition (EMT). TGF- $\beta$ receptor protein 1 (T $\beta R 1)$ is an important component of the TGF- $\beta$ signaling pathway (53). Researchers showed that SIRT7 depletion decreases T $\beta R 1$ levels and reduces downstream signaling. The effect on T $\beta R 1$, however, was indirect as SIRT7 did not interact with T $\beta R 1$, but the mediator PICK1 (protein interacting with protein kinase $\mathrm{C}$, alpha), which interacts with T $\beta \mathrm{R} 1$ and SIRT7 together. This study also showed that loss of SIRT7 activates autophagy and PICK1, again affecting T $\beta$ RI status. Thus, the researchers concluded that SIRT7 maintains T $\beta$ RI protein levels by modulating autophagy and PICK1 to regulate the TGF- $\beta$ signaling pathway. In this way, SIRT7 can participate in scar formation, angiogenesis, inflammation and wound healing in response to acute cardiovascular injury (52). Based on these data, SIRT7 may be considered as a good predictor or therapeutic target for cardiac diseases.

\section{Genome Stability}

Sirtuins, including SIRT7, maintain genomic stability under stress conditions through a variety of mechanisms $(24,31,54,55)$. SIRT7 protects the genome largely by influencing chromatin structure, cell-cycle progression and DNA damage signaling and repair (Figure 3). SIRT7 knockout mice show an aging-like phenotype, associated with an increased sensitivity to oxidative 
A Chromatin regulation

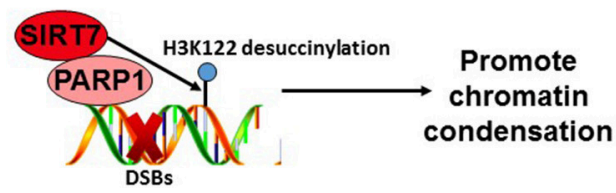

B Cell cycle

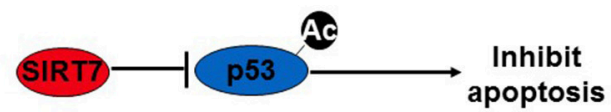

\section{DNA damage and repair}
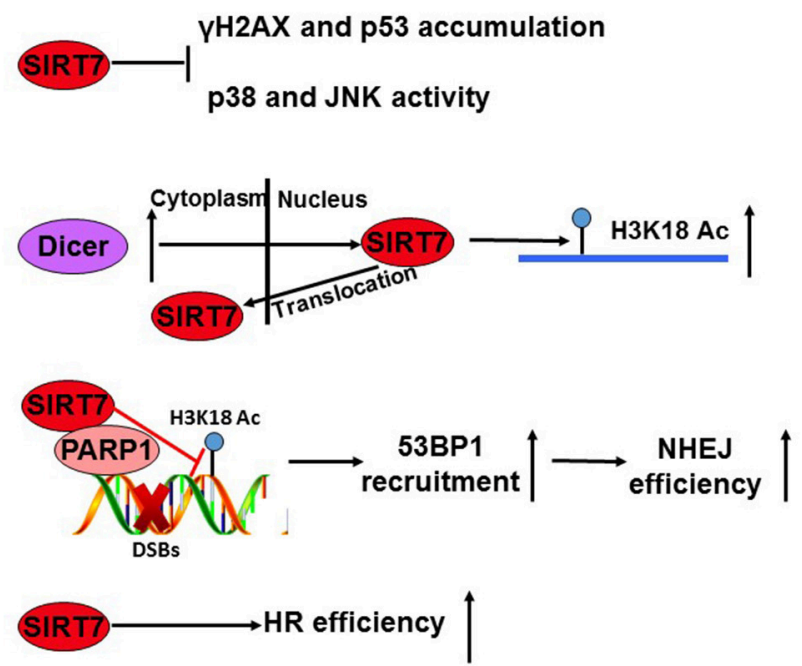

FIGURE 3 | SIRT7 maintains genome stability through multiple pathways. Under stress conditions, SIRT7 participates in the maintenance of genome stability via multiple mechanisms. (A) SIRT7 is recruited to DSBs in a PARP1-dependent manner to catalyze desuccinylation of H3K122 at DSBs to promote chromatin condensation. (B) SIRT7 can deacetylate p53 to inhibit apoptosis. (C) SIRT7 exerts a protective role during genomic insults by attenuating the DNA damage response (preventing $\gamma \mathrm{H} 2 \mathrm{AX}$ and p53 accumulation) and the stress-activated MAPK pathway (p38 and JNK activity inhibition). The upregulation of Dicer in cytoplasm promotes SIRT7 translocation from the nucleus to the cytoplasm, thereby causing a decrease in chromatin-associated SIRT7 and elevated H3K18Ac levels. Besides, SIRT7 overexpression increases the efficiency of non-homologous end joining (NHEJ) and homologous recombination (HR). The recruitment of SIRT7 to sites of DNA damage by a PARP1-dependent manner also modulates H3K18Ac levels, thereby influencing 53BP1 recruitment to DNA double-strand breaks (DSBs) to increase the efficiency of NHEJ.

and genotoxic stress, suggesting a link between SIRT7 and genomic protection (8).

SIRT7 can maintain genomic stability by regulating p53 or DNA damage repair. With regards to p53, Vakhrusheva et al. found that SIRT7 knockout mouse embryonic fibroblasts (MEFs) undergo higher levels of apoptosis than control MEFs upon adriamycin treatment. This process is as a result of p53 hyperacetylation in the absence of SIRT7 (8). Kiran et al. found that SIRT7 exerts a protective role during genomic insults by attenuating the DNA damage response (preventing $\gamma \mathrm{H} 2 \mathrm{AX}$ and p53 accumulation) and the stress-activated MAPK pathway (p38 and JNK activity inhibition) (31). Moreover, they found that SIRT7 transfers from the nucleolus to the nuclear matrix after doxorubicin chemotherapy (31). Similarly, Zhang et al. found that SIRT7 translocates from the nucleus to the cytoplasm in response to DNA damage. This translocation occurs through an interaction with Dicer, causing a decrease in chromatinassociated SIRT7 and elevated H3K18Ac levels (56).

Regarding DNA damage repair, Mao et al. found that SIRT7 overexpression increases the efficiency of non-homologous end joining (NHEJ) by 1.5-fold and homologous recombination (HR) by 2.8 -fold in paraquat toxin-treated human fibroblast cells (57). A more recent study by Vazquez et al. elucidated a mechanism by which SIRT7 contributes to NHEJ. They demonstrated that SIRT7 is recruited to sites of DNA damage in a PARP1-dependent manner, where it modulates H3K18Ac levels, thereby influencing $53 \mathrm{BP} 1$ recruitment to DNA double-strand breaks (DSBs) to increase the efficiency of NHEJ (55). These data provide direct evidence for a role of SIRT7-mediated H3K18 deacetylation in maintaining genome integrity by DSB repair. At the same time, Li et al. also found that SIRT7 is recruited to DSBs in a PARP1dependent manner, but showed that it catalyzes desuccinylation of $\mathrm{H} 3 \mathrm{~K} 122$ at DSBs to promote chromatin condensation and efficient DSB repair (24). These latest findings extend our understanding as to how SIRT7 helps maintain genome stability.

\section{Metabolic Regulation Glucose Metabolism}

The enzymatic activity of SIRT7 depends on its metabolic cosubstrate $\mathrm{NAD}^{+}$, thus connecting its role to cellular metabolic status. SIRT7 is a low glucose stress sensor that, as discussed, modulates rDNA transcription to preserve energy and resist nutritional stress. Chen et al. identified that SIRT7 redistributes from the nucleoli to the nucleoplasm upon glucose deprivation or treatment with AICAR (a low energy mimic) (12). At the mechanistic level, PAF53 interacts with Pol I and recruits it to rDNA promoters (58). SIRT7 deacetylates PAF53, which acts as a signal to recruit RNA polymerase I to rDNA promoters and activate RNA Pol I-mediated transcription. Under low glucose conditions, the redistribution of SIRT7 from the nucleoli to the nucleoplasm permits PAF53 hyper-acetylation. This hyper-acetylation impairs the interaction between PAF53 and Pol I and decreases Pol I activity, thus leading to rDNA transcription inhibition (12). Sun et al. also showed that glucose 
starvation induces SIRT7 redistribution via AMPK-directed SIRT7 phosphorylation. This effect causes REG $\gamma$-proteasomedependent degradation, thereby reducing rDNA transcription to avoid cell death (44).

Recent studies have provided insight on the role of SIRT7 in glycolysis. SIRT7 deacylates phosphoglycerate kinase 1 (PGK1), a key enzyme in glycolysis pathway, and suppresses PGK1 enzymatic activity in liver cancer cells (15). We also reported that SIRT7 regulates gluconeogenesis by modulating G6PC expression via USP7-mediated deubiquitination (45). SIRT7 also suppresses HIF1 and HIF2, which repress glucose oxidation through the tricarboxylic acid cycle (59). Taken together, these findings implicate SIRT7 in glucose metabolism.

\section{Lipid Metabolism}

The evidence supporting a role for SIRT7 in lipid metabolism in the liver is conflicting (60). Yoshizawa et al. found that SIRT7 knockout mice (generated by deleting exons 4-9), are resistant to high-fat diet (HFD)-induced fatty liver, glucose intolerance and obesity (61). They also showed that liverspecific SIRT7 knockout mice have reduced hepatic triglyceride accumulation (61). SIRT7 activates Cd36 expression, which is vital for fatty-acid uptake, as well as Mogat that incorporates fatty acids into triglycerides, and Cidea and Cidec that are involved in lipid storage and lipid droplet formation. The expression levels of these four genes were all reduced in the livers of the SIRT7 knockout mice fed a HFD (61). Mechanistically, an interaction between the E3 ubiquitin ligase complex (DDB1-CUL4-associated factor 1 (DCAF1)/damagespecific DNA binding protein 1 (DDB1)/cullin 4B (CUL4B) complex) and TR4 (a nuclear receptor involved in lipid metabolism) promotes TR4 degradation. However, SIRT7 binding to the DCAF1/DDB1/CUL4B complex inhibits TR4 degradation and activates TR4 target genes to increase fatty-acid uptake and triglyceride synthesis and storage. Consequently, the expression level of TR4 and its target genes are reduced in liverspecific SIRT7 knockout mice and lipid synthesis and storage is decreased to resist hepatic steatosis (61).

Two other groups have reported opposing results to Yoshizawa et al. finding that SIRT7 knockout mice instead suffer hepatic steatosis $(13,38)$. Shin et al. found that SIRT7 knockout mice (generated by replacing exons $4-11$ with a LacZ gene), have a fatty liver without obesity (38). They showed that loss of SIRT7 increases lipogenic gene expression, liver triglyceride levels and inflammatory markers, indicating progression to steatohepatitis. These mice also exhibited low levels of plasma triglycerides compared to wild-type controls, due to reduced very-low-density lipoprotein (VLDL) secretion. Importantly, liver steatosis was reversed in these animals by reintroducing SIRT7 specifically in the liver (38). At the mechanistic level, it seems that SIRT7 prevents the development of fatty liver disease by suppressing ER stress. Consequently, SIRT7 knockout mice fail to relieve ER stress such that the UPR pathway is activated causing apoptosis, inflammation, increased lipogenesis and reduced VLDL secretion specifically in the liver (38).

Ryu et al. also described that SIRT7 knockout mice (generated by deleting exons 6-9) exhibited hepatic microvesicular steatosis, and increased plasma levels of triglycerides and free fatty acids. These mice also showed signs of multi-systemic mitochondrial dysfunction due to GABP $\beta 1$ hyperacetylation in the absence of SIRT7, including increased blood lactate levels and reduced exercise performance. As discussed, SIRT7 mediates mitochondrial function by deacetylating GABP $\beta 1$ (a regulator of multiple nuclear-encoded mitochondrial genes) to promote the formation and activation of the GABP complex that induces mitochondrial gene expression and contributes to mitochondrial homeostasis (13).

Although they used the same mice strain (C57BL/6J), the contradiction of the roles of SIRT7 in lipid metabolism discussed in these three studies may be due to the differences in the genetic background of and construction of SIRT7 knockout mice (60). Further studies with more sample size and parallel experiments are required to clarify this picture.

Cioffi et al. found that SIRT7 induces differentiation and maturation of early adipocyte precursors to promote adipogenesis (39). Consistently, SIRT7 knockdown resulted in reduced Oil Red $\mathrm{O}$ staining and adipogenesis marker (FABP4, PPAR $\gamma, \mathrm{C} / \mathrm{ebp} \alpha$, adipoq) expression. The researchers also found that miR-93 prevents adipogenesis by inhibiting SIRT7: adipogenesis was enhanced in mir-25-93-106 $\mathrm{b}^{-/-}$mice but repressed in miR-93-reintroduced mice. Interestingly, SIRT7 expression was enhanced in the mir-25-93-106 $\mathrm{b}^{-/-}$mice while nuclear SIRT7 expression was reduced upon injection of miR93 mimics into the visceral fat pads of leptin-deficient (ob/ob) mice (39). Despite these preliminary findings, the definitive mechanism underlying how miR-93 regulates SIRT7 and how SIRT7 promotes adipogenesis remains unclear. Fang et al. demonstrated that SIRT7 can promote adipogenesis by binding to SIRT1 and inhibiting its activity by preventing its autodeacetylation. SIRT1 is reported to repress PPAR $\gamma$ through interaction with nuclear receptor corepressor 1 (NCoR1, PPAR $\gamma$ corepressor) to inhibit adipogenesis (62). As such, SIRT7 knockout mice possess a notably diminished proportion of white fat due to enhanced SIRT1 activity, which blocks PPAR $\gamma$ and adipocyte differentiation (63). SIRT1 activity depletion restores adipogenesis in Sirt7 knockout mice (63). These data implicate a potential cross-regulatory network within the sirtuin family.

\section{SIRT7 in Mitochondrial Metabolism}

As discussed, the mitochondrion is a crucial organelle involved in regulating cellular energy homeostasis and thus cell survival. Mitochondria uptake energy from nutrients and then convert it into ATP by oxidative phosphorylation (OXPHOS) (64). In response to cellular stress, the mitochondrion has an armamentarium of quality-control mechanisms based on mitochondrial biosynthesis, mitophagy and mitochondrial unfolded protein responses, to maintain proper mitochondrial function. SIRT7 is an important regulator of mitochondrial homeostasis. As stated above, Ryu et al. found that SIRT7 knockout mice show multi-systemic mitochondrial dysfunction, including lactate accumulation in the blood and age-related hearing loss (13). Mechanistically, SIRT7 impacts on mitochondria function by deacetylating GABP $\beta 1$, which forms a hetero-tetramer complex with $\mathrm{GABP} \alpha$, occupies 


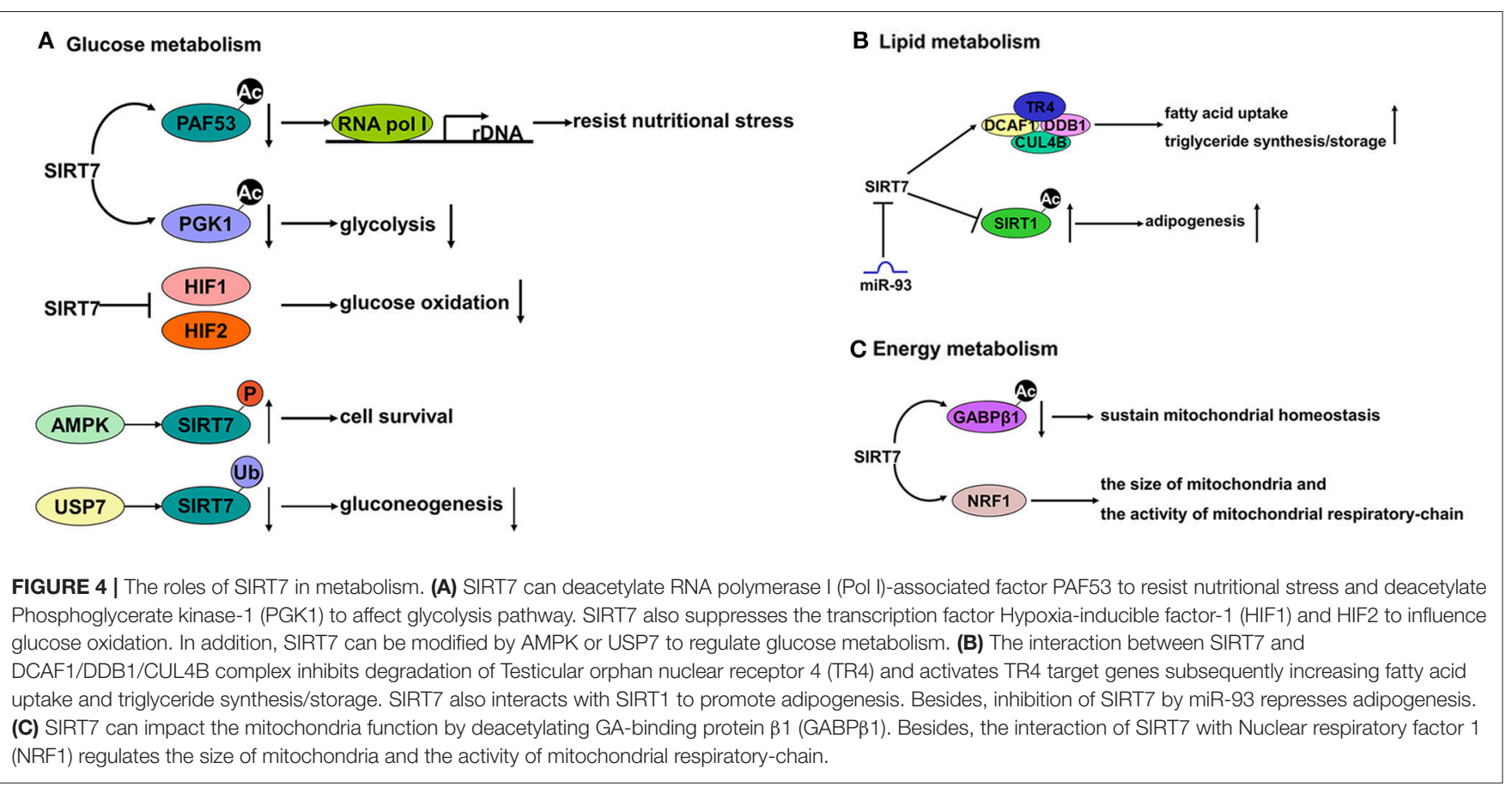

nuclear-encoded mitochondrial target genes and promotes their transcription. Further studies also found that SIRT7 deficiency affects OXPHOS in the heart and liver (13). Mohrin et al. also reported that SIRT7 interacts with NRF1 (a master regulator of mitochondria) to regulate mitochondria size and activity of the mitochondrial respiratory-chain (46).

Overall, SIRT7 has a vital role in metabolic homeostasis (Figure 4), but there remain many unresolved questions. The contradictory effect of SIRT7 on lipid metabolism also needs clarification. Future studies need to address these issues and determine whether SIRT7 may be a suitable therapeutic target in metabolic disorders.

\section{Aging and Senescence}

Consistent with the known functions of sirtuins in senescence and lifespan-extension, numerous studies have established a close relationship between SIRT7 and age-related processes (36). SIRT7-knockout mice have a shorter lifespan than control mice, and succumb to premature aging phenotypes around 1 year, with symptoms, such as kyphosis, decreased gonadal fat pad content, reduced IGF-1 plasma levels, hepatic steatosis, degenerative heart hypertrophy, reduced hearing and reduced hematopoietic stem cell-regenerative potential $(13,46,65)$. SIRT7 expression gradually declines with age in mice, rat and several cells, including endothelial cells, fibroblasts, hepatocytes and HEK293FT cells $(13,14,66-68)$. Kiran et al. found that SIRT7 over-expressing cells show a mostly normal morphology, with very few enlarged cells upon treatment with a low dose of doxorubicin (a cellular senescence inducing agent) (69). By contrast, control GFP expressing cells exhibit cell enlargement and multi-nucleation (typical features of cellular senescence) following doxorubicin exposure. The researchers also found that p53 and p21 senescence marker expression decreases in SIRT7 overexpressing cells upon doxorubicin treatment compared to control cells.

Lee et al. reported that the acetylation levels of nucleophosmin (NPM1) are increased while SIRT6 and SIRT7 levels are decreased in senescent cells. SIRT7 can deacetylate NPM1, which results in up-regulated p53 transcriptional activity in MEFs to induce cellular senescence $(14,70)$.

Telomeres have the potential to serve as the biomarker of biological cell age (71). Conomos et al. found that TR4 recruitment to the telomere can attribute to the ALT (alternative lengthening of telomeres) phenotype (72). SIRT7 positively regulates the protein level of TR4 (19), suggesting that SIRT7 might play an upstream role in DNA repair and telomere maintenance pathways.

A role for SIRT7 in aging and senescence has also been attributed to SIRT7-mediated regulation of mitochondrial ribosomal proteins (mRPs). SIRT7 interacts with NRF1 to repress mRPs, resulting in hematopoietic stem-cell longevity (46). Conversely, SIRT7-mediated GABP $\beta 1$ deacetylation promotes the formation and activation of the GABP complex to increase mRPs, resulting in hematopoietic stem-cell aging (13).

One study reported an interaction between SIRT7 with Tripeptidyl peptidase II (TPPII) that permits SIRT7 cytoplasmic localization (52). TPPII has regulatory effects on apoptosis and senescence, as observed in TPPII knockout mice that exhibit early immuno-senescence and have a shorter lifespan than controls (73). SIRT7 may, therefore, regulate aging and senescence by interacting with TPPII, but the underlying mechanisms need further study.

rDNA instability is common in premature aging syndromes. A recent study by Paredes et al. uncovered a critical role for 

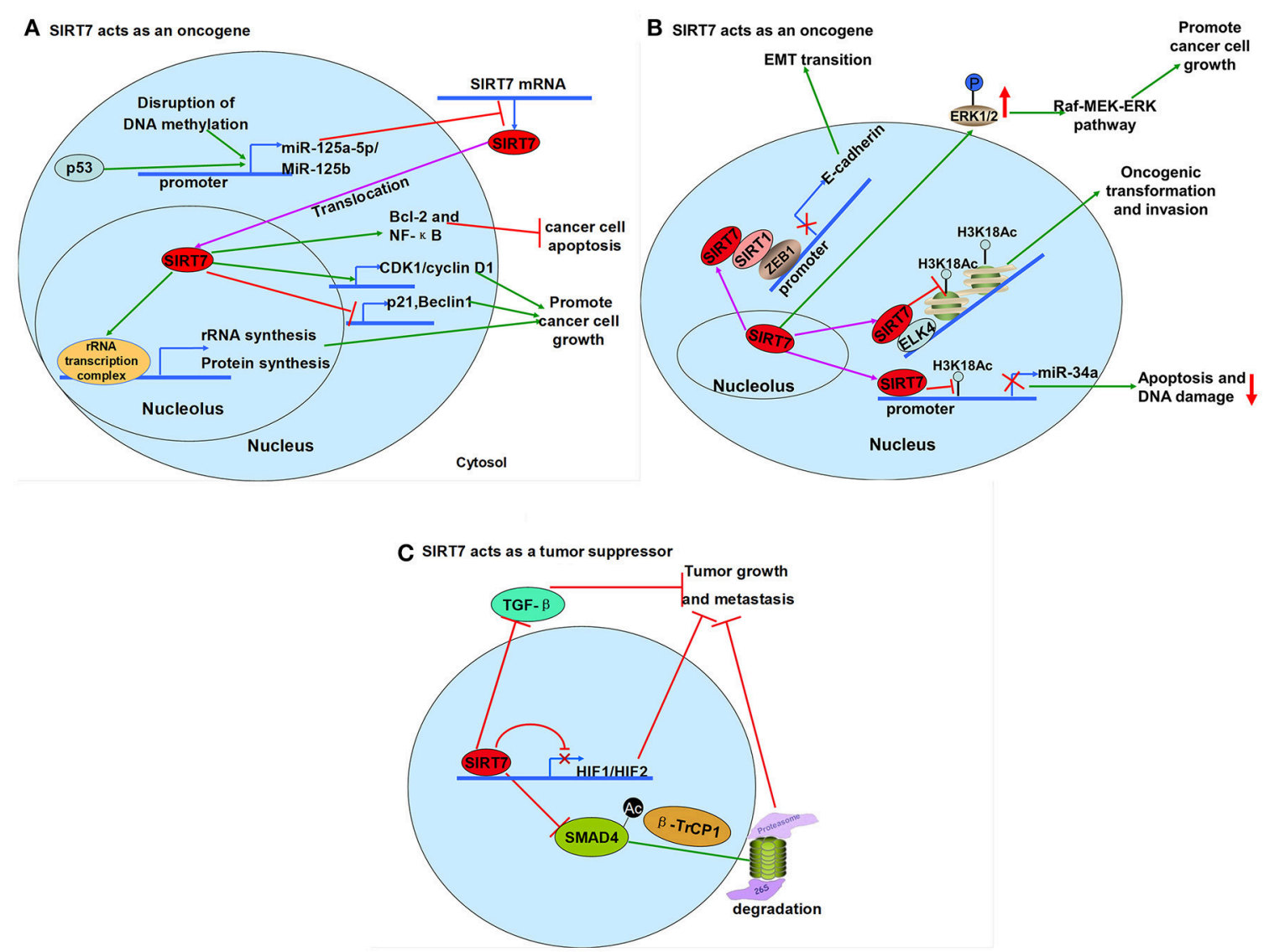

FIGURE 5 | The roles of SIRT7 in cancer. (A,B) SIRT7 acts as an oncogene. (A) The disruption of DNA methylation and p53 activates miR-125b and miR-125a-5p to inhibit the expression of SIRT7. Overexpression of SIRT7 upregulates the expression of CDK1/cyclin D1 while downregulates the level of p21 and Beclin1 as well as promotes rRNA and protein synthesis by interaction with rRNA transcription complex to promote cancer cell growth. In addition, overexpression of SIRT7 can also upregulate Bcl-2 and NF-kB to repress cancer cell apoptosis. (B) ETS-like transcription factor 4 (ELK4) targets SIRT7 to promoters of many tumor suppressor genes for H3K18 deacetylation and leads to cancer cell growth and metastasis eventually. SIRT7 induces ERK1/2 phosphorylation and activated the Raf-MEK-ERK pathway to promote cancer cell growth. SIRT7 also reduces miR-34a expression by deacetylating H3K18Ac to decrease cancer cell apoptosis and DNA damage. Besides, SIRT7 reduces expression of E-cadherin by interaction with SIRT1 to promote EMT transition. (C) SIRT7 acts as a tumor suppressor. SIRT7 inactivates TGF- $\beta$ signaling and represses epithelial-to-mesenchymal transition. SIRT7 also negatively regulates HIF1 and HIF2 transcription. Moreover, SIRT7 deacetylates Mothers against decapentaplegic homolog 4 (SMAD4) and promotes $\beta$-transducin repeat-containing protein 1 ( $\beta$-TrCP1)-dependent degradation to inhibit tumor growth and metastasis.

SIRT7 in protecting against cellular senescence by maintaining heterochromatin silencing and rDNA stability. Mechanistically, they found that SIRT7 acts as a scaffold to stabilize SNF2H at rDNA promoters for chromatin silencing (74). Taken together, SIRT7 might prevent aging-induced physiological changes and possibly extend lifespan via numerous pathways.

\section{Cancer}

Although ample evidence supports the involvement of SIRT7 in carcinogenesis, SIRT7 exhibits opposing roles in different cancer types (Figure 5). SIRT7 is up- regulated in the majority of cancers, including colorectal, gastric, thyroid, node-positive breast, bladder, ovarian and cervical cancers, hepatocellular and epithelial prostate carcinoma, where it acts as an oncogene $(9,40$, 75-82). The exception is seen in pancreatic cancer, where SIRT7 is down-regulated and seems to act as a tumor suppressor (83) (Table 2).

As an oncogene, SIRT7 inhibition reduces cancer-cell growth, represses colony formation and cancer-cell metastasis and increases cancer-cell apoptosis. High SIRT7 expression is also considered a predictor of poor survival in various cancers (9, 40, 75-82). Many studies have reported mechanisms of action by which SIRT7 promotes cancer-cell growth (Figure 5). $\mathrm{Yu}$ et al. found that SIRT7 induces ERK1/2 phosphorylation and activates the Raf-MEK-ERK pathway to promote cancercell growth (81). Hypoacetylation of H3K18 is associated with oncogenic transformation, aggressive tumor phenotypes and poor prognosis and maintenance of essential human cancer-cell features, including anchorage-independent growth and escape from contact inhibition (84). As shown in prostate cancer, SIRT7 binds and maintains the deacetylated state of H3K18 at 
TABLE 2 | SIRT7 related cancers.

\begin{tabular}{lll}
\hline Cancer & $\begin{array}{l}\text { SIRT7 } \\
\text { expression }\end{array}$ & Role/Impact \\
\hline Colorectal cancer & Overexpression & Oncogene \\
Colorectal cancer & Downregulated & $\begin{array}{l}\text { Induce radio-sensitivity; } \\
\text { Enhance therapeutic effects }\end{array}$ \\
Gastric cancer & Overexpression & Oncogene \\
Thyroid cancer & Overexpression & Oncogene \\
Node-positive & Overexpression & Oncogene \\
breast cancer & & \\
Breast cancer & Deficiency & Increase metastasis \\
Bladder cancer & Overexpression & Oncogene; \\
Ovarian cancer & Overexpression & Oncogene \\
Cervical cancer & Overexpression & Oncogene \\
Hepatocellular & Overexpression & Oncogene; \\
carcinoma & & promote cancer-cell growth \\
Epithelial prostate & Overexpression & Oncogene; \\
cacinoma & maintaining cancer phenotypes; \\
Pancreatic cancer & Downregulated & increase metastasis \\
Liver cancer & Knockdown & Promote cancer-cell growth \\
& &
\end{tabular}

the promoters of many tumor suppressor genes by interacting with the ELK4 transcription factor $(85,86)$. Thus, SIRT7 has a fundamental role in maintaining cancer phenotypes (6).

Zhang et al. found that SIRT7 knockdown promotes gastric cancer-cell apoptosis (82). Mechanically, SIRT7 prevents cellular apoptosis by down-regulating miR-34a via $\mathrm{H} 3 \mathrm{~K} 18 \mathrm{ac}$ deacetylation. The miR-34 family is associated with cell-cycle arrest, senescence and apoptosis in cancers, and low miR-34a expression is associated with poor prognosis (82).

Another mechanism as to how SIRT7 promotes cancercell growth was identified by Kim et al. (9). They found that SIRT7 knockdown increases the number of liver cancer cells in G1/S phase and delays the cell-cycle transition: p21 WAF1/Cip1 expression was increased and cyclin D1 (a G1/S cell cycle regulator) was suppressed in SIRT7 knockdown cells. In patients with hepatocellular carcinoma, increased SIRT7 expression was attributed to $\mathrm{p} 53$ mutation or the endogenous hypermethylation of the microRNAs miR-125a-5p and miR-125b. Over-expression of SIRT7 led to $\mathrm{p} 21^{\mathrm{WAF} 1 / \mathrm{Cip} 1}$ suppression and induction of cyclin D1 expression to promote cancer-cell growth (9). Han et al. also found that SIRT7 expression is regulated by hsa-miR-125b in bladder cancer. They observed that in bladder cancer, hsa-miR$125 \mathrm{~b}$ and SIRT7 are inversely associated with the oncogenic long non-coding RNA MALAT1. Up-regulated hsa-miR-125b resulted in down-regulated SIRT7 and MALAT1; this effect inhibited bladder cancer cell growth, induced apoptosis, and decreased cell motility (40).

With regards to apoptosis, Wang et al. found that shRNAmediated SIRT7 silencing reduced anti-apoptotic factor B-cell lymphoma 2 (Bcl-2) and nuclear factor kappa B (NF- $\kappa$ B) levels (80). The NF- $\kappa B$ signaling pathway is important in cellular proliferation, apoptosis and migration in malignant diseases
(87). The researchers concluded that SIRT7 inhibits cancer-cell apoptosis by up-regulating Bcl-2 and NF- $\kappa \mathrm{B}$ levels (80). The precise regulatory mechanisms remain to be identified.

SIRT7 also influences cancer-cell metastasis. High SIRT7 expression is associated with aggressive cancer phenotypes, metastatic diseases and poor patient prognosis; down-regulating SIRT7 reverses the metastatic properties of epithelial and mesenchymal cancer cells. In epithelial carcinomas, SIRT7 is associated with the EMT, a key process in metastatic progression. Malik et al., Yu et al., Zhang et al. focused on classic EMT regulatory factors and discovered that the expression of $\mathrm{E}$ cadherin (an EMT regulatory factor) showed inverse correlation with SIRT7 in vivo. In SIRT7-deficient cells, E-cadherin and DAB2 interacting protein (DAB2IP; a tumor suppressor gene, whose loss promotes EMT and metastasis in prostate cancer) are significantly increased in mRNA level. These findings suggest a role for SIRT7 in cancer prevention and as prognostic factor (78, 81, 82). SIRT7 interacts with SIRT1 to enhance SIRT1-dependent prostate cancer cell metastatic properties and promotes Ecadherin transcriptional repression (88).

In non-epithelial cancers, SIRT7 may impact metastasis regulatory pathways to affect cellular metastatic properties. The expression levels of matrix metalloproteinase MMP16 and vascular endothelial growth factor (VEGF-A) are reduced in SIRT7-deficient HT1080 cells compared to control cells (78). Thus, SIRT7 may be of vital importance for cancer-cell metastasis (78).

As a tumor suppressor, low levels of SIRT7 are associated with aggressive tumor phenotypes and poor patient outcomes. One study found that patients with pancreatic cancer and high levels of nuclear SIRT7 had a longer lifespan (succumbed to disease later) than those with low levels of SIRT7. However, the precise mechanism underlying this association is unclear (83). Similar to the other sirtuins, SIRT7 has also been recognized as a tumor suppressor based on its negative regulation of HIF1 and HIF2 transcription $(50,89)$, as previously discussed.

The contradictory roles of SIRT7 in cancer may be related to its multiple interactions and functions in various cellular processes. First, SIRT7 deacetylates H3K18Ac, whose depletion is associated with highly malignant cancers and poor patient prognosis. Second, SIRT7 influences ribosome biogenesis to meet the high biosynthetic and metabolic needs of cancer cells. At the same time, although SIRT7 inactivates p53 by deacetylation, up to half of all tumors exhibit mutated p53, which may diminish the oncogenic role of SIRT7 and result in tumor suppressive characteristics $(83,90)$. Even though the definitive function of SIRT7 is uncertain, many consider SIRT7 as a cancer biomarker or a predictor of prognosis $(73,75,81,83)$. In sum, data suggest that SIRT7 may be a potential novel biomarker for prognosis in pancreatic cancer (83), a circulating marker in head and neck squamous cell carcinoma (73), and a predictive biomarker of pancreatic cancer (PCa) aggressiveness (91).

Our lab also found that down-regulation of SIRT7 after 5 -FU exposure induces radio-sensitivity in human colorectal cancer and enhances therapeutic effects (92). Shi et al. found the microRNA-3666 induces SIRT7 inhibition, which in turn inhibits non-small cell lung cancer cell growth (41). 
Recently, Tang et al. found that SIRT7 deficiency promotes breast cancer cell metastasis, while temporal expression of SIRT7 inhibits metastasis in a polyomavirus middle $\mathrm{T}$ antigen breast cancer model. Here, SIRT7 deacetylates SMAD4 and promotes $\beta$-TrCP1-dependent degradation. Finally, SIRT7 deficiency activates TGF- $\beta$ signaling and enhances the EMT (21). Therefore, it can be assumed that SIRT7 is a worthwhile target to explore for cancer therapy.

\section{CONCLUSIONS AND FUTURE PERSPECTIVES}

The function of SIRT7 was ignored in the initial years of sirtuinbased research due to its localization in the nucleolus. However, major breakthroughs have been made over the past decade in SIRT7 biology. New substrates for SIRT7 deacetylase activity have been identified, which have highlighted new enzymatic activities that are critical for different cellular processes. SIRT7 has strong potential as a therapeutic target in various cancers, due to the identification of its up-regulation and activation in various cancer cells. SIRT7 is also considered a potential novel biomarker for prognosis in several cancers, such as pancreatic cancer. However, due to its complicated and controversial mechanism in the maintenance of cancers, future studies are needed to understand the precise molecular mechanism and downstream pathways of SIRT7 in the specific cancer types. The therapeutic uses of SIRT7 in cancers will rely on the further clinical trials.

\section{REFERENCES}

1. Imai S, Armstrong CM, Kaeberlein M, Guarente L. Transcriptional silencing and longevity protein Sir2 is an NAD-dependent histone deacetylase. Nature (2000) 403:795. doi: 10.1038/35001622

2. Landry J, Sutton A, Tafrov ST, Heller RC, Stebbins J, Pillus L, et al. The silencing protein SIR2 and its homologs are NAD-dependent protein deacetylases. Proc Natl Acad Sci USA. (2000) 97:5807-11. doi: 10.1073/pnas.110148297

3. Michishita E, Park JY, Burneskis JM, Barrett JC, Horikawa I. Evolutionarily conserved and nonconserved cellular localizations and functions of human SIRT proteins. Mol Biol Cell (2005) 16:4623-35. doi: 10.1091/mbc.e05-01-0033

4. Haigis MC, Sinclair DA. Mammalian sirtuins: biological insights and disease relevance. Annu Rev Pathol. (2010) 5:253-95. doi: 10.1146/annurev.pathol.4.110807.092250

5. Voelter-Mahlknecht S, Letzel S, Mahlknecht U. Fluorescence in situ hybridization and chromosomal organization of the human sirtuin 7 gene. Int J Oncol. (2006) 28:899-908. doi: 10.3892/ijo.28.4.899

6. Barber MF, Michishita-Kioi E, Xi Y, Tasselli L, Kioi M, Moqtaderi $\mathrm{Z}$, et al. SIRT7 links H3K18 deacetylation to maintenance of oncogenic transformation. Nature (2012) 487:114-8. doi: 10.1038/nature 11043

7. Tsai Y, Greco TM, Boonmee A, Miteva Y, Cristea IM. Functional proteomics establishes the interaction of SIRT7 with chromatin remodeling complexes and expands its role in regulation of RNA polymerase I transcription. $\mathrm{Mol}$ Cell Proteomics (2011) 11:60-76. doi: 10.1074/mcp.A111.015156

8. Vakhrusheva O, Smolka C, Gajawada P, Kostin S, Boettger T, Kubin T, et al. SIRT7 increases stress resistance of cardiomyocytes and prevents apoptosis and inflammatory cardiomyopathy in mice. Circ Res. (2008) 102:703-10. doi: 10.1161/CIRCRESAHA.107.164558
A recent report suggests a role for SIRT7 in the adaptive immune system and neurogenesis (93). This finding indicates that the roles and function of SIRT7 are still diversifying, and are wider than previously thought. Further studies are now needed to better understand and elucidate the molecular role of SIRT7, identify its substrate partners/cofactors, and delineate the intracellular pathways that regulate their activity in different disease models.

\section{AUTHOR CONTRIBUTIONS}

$\mathrm{DW}, \mathrm{YL}$, and $\mathrm{KZ}$ wrote the primary manuscript and revised the manuscript. HW and W-GZ conceived and designed the manuscript.

\section{FUNDING}

This review was supported by the National Natural Science Foundation of China (grant number 81472627), the Discipline Construction Funding of Shenzhen (2016), and the Shenzhen Municipal Commission of Science and Technology Innovation (grant number JCYJ20160427104855100).

\section{ACKNOWLEDGMENTS}

The authors would like to thank Dr. Jessica Tamanini (Shenzhen University and ETediting) for editing the manuscript prior to submission.
9. Kim JK, Noh JH, Jung KH, Eun JW, Bae HJ, Kim MG, et al. Sirtuin7 oncogenic potential in human hepatocellular carcinoma and its regulation by the tumor suppressors MiR-125a-5p and MiR-125b. Hepatology (2013) 57:1055-67. doi: 10.1002/hep.26101

10. Nahalkova J. Novel protein-protein interactions of TPPII, p53, and SIRT7. Mol Cell Biochem. (2015) 409:13-22. doi: 10.1007/s11010-015-2507-y

11. Chen S, Blank MF, Iyer A, Huang B, Wang L, Grummt I, et al. SIRT7dependent deacetylation of the U3-55k protein controls pre-rRNA processing. Nat Commun. (2016) 7:10734. doi: 10.1038/ncomms10734

12. Chen S, Seiler J, Santiago-Reichelt M, Felbel K, Grummt I, Voit R. Repression of RNA polymerase I upon stress is caused by inhibition of RNAdependent deacetylation of PAF53 by SIRT7. Mol Cell (2013) 52:303-13. doi: 10.1016/j.molcel.2013.10.010

13. Ryu D, Jo YS, Lo Sasso G, Stein S, Zhang H, Perino A, et al. A SIRT7-dependent acetylation switch of GABP $\beta 1$ controls mitochondrial function. Cell Metab. (2014) 20:856-69. doi: 10.1016/j.cmet.2014.08.001

14. Lee N, Kim D, Kim E, Park SJ, Kwon J, Shin J, et al. Comparative interactomes of SIRT6 and SIRT7: implication of functional links to aging. Proteomics (2014) 14:1610-22. doi: 10.1002/pmic.201400001

15. $\mathrm{Hu} \mathrm{H}$, Zhu W, Qin J, Chen M, Gong L, Li L, et al. Acetylation of PGK1 promotes liver cancer cell proliferation and tumorigenesis. Hepatology (2016) 65:515-28. doi: 10.1002/hep.28887

16. Yu J, Qin B, Wu F, Qin S, Nowsheen S, Shan S, et al. Regulation of serine/threonine kinase Akt activation by NAD (+)-dependent deacetylase SIRT7. Cell Rep. (2017) 18:1229-40. doi: 10.1016/j.celrep.2017.01.009

17. Blank MF, Chen S, Poetz F, Schnölzer M, Voit R, Grummt I. SIRT7-dependent deacetylation of CDK9 activates RNA polymerase II transcription. Nucleic Acids Res. (2017) 45:2675-86. doi: 10.1093/nar/gkx053

18. Li Z, Bridges B, Olson J, Weinman SA. The interaction between acetylation and serine-574 phosphorylation regulates the apoptotic function of FOXO3. Oncogene (2016) 36:1887-98. doi: 10.1038/onc.2016.359 
19. Karim MF, Yoshizawa T, Sobuz SU, Sato Y, Yamagata K. Sirtuin 7dependent deacetylation of DDB1 regulates the expression of nuclear receptor TR4. Biochem Biophys Res Commun. (2017) 490:423-8. doi: 10.1016/j.bbrc.2017.06.057

20. Mo Y, Lin R, Liu P, Tan M, Xiong Y, Guan K, et al. SIRT7 deacetylates DDB1 and suppresses the activity of the CRL4 E3 ligase complexes. FEBS J. (2017) 284:3619-36. doi: 10.1111/febs.14259

21. Tang X, Shi L, Xie N, Liu Z, Qian M, Meng F, et al. SIRT7 antagonizes TGF- $\beta$ signaling and inhibits breast cancer metastasis. Nat Commun. (2017) 8:318. doi: 10.1038/s41467-017-00396-9

22. Song C, Hotz-Wagenblatt A, Voit R, Grummt I. SIRT7 and the DEAD-box helicase DDX21 cooperate to resolve genomic R loops and safeguard genome stability. Gene Dev. (2017) 31:1370-81. doi: 10.1101/gad.300624.117

23. Zhang C, Zhai Z, Tang M, Cheng Z, Li T, Wang H, et al. Quantitative proteome-based systematic identification of SIRT7 substrates. Proteomics (2017) 17:1600395. doi: 10.1002/pmic.201600395

24. Li L, Shi L, Yang S, Yan R, Zhang D, Yang J, et al. SIRT7 is a histone desuccinylase that functionally links to chromatin compaction and genome stability. Nat Commun. (2016) 7:12235. doi: 10.1038/ncomms12235

25. Tsai Y, Greco TM, Cristea IM. Sirtuin 7 plays a role in ribosome biogenesis and protein synthesis. Mol Cell Proteomics (2013) 13:73-83. doi: $10.1074 / \mathrm{mcp} . M 113.031377$

26. Tanabe K, Liu J, Kato D, Kurumizaka H, Yamatsugu K, Kanai M, et al. LC-MS/MS-based quantitative study of the acyl group- and siteselectivity of human sirtuins to acylated nucleosomes. Sci Rep. (2018) 8:2656. doi: 10.1038/s41598-018-21060-2

27. Tong Z, Wang M, Wang Y, Kim DD, Grenier JK, Cao J, et al. SIRT7 is an RNA-activated protein lysine deacylase. ACS Chem Biol. (2016) 12:300-10. doi: 10.1021/acschembio.6b00954

28. Tong Z, Wang Y, Zhang X, Kim DD, Sadhukhan S, Hao Q, et al. SIRT7 is activated by DNA and deacetylates histone $\mathrm{H} 3$ in the chromatin context. ACS Chem Biol. (2016) 11:742-7. doi: 10.1021/acschembio.5b01084

29. Karim MF, Yoshizawa T, Sato Y, Sawa T, Tomizawa K, Akaike T, et al. Inhibition of H3K18 deacetylation of SIRT7 by Myb-binding protein 1a (Mybbp1a). Biochem Biophys Res Commun. (2013) 441:157-63. doi: 10.1016/j.bbrc.2013.10.020

30. Ford E, Voit R, Liszt G, Magin C, Grummt I, Guarente L. Mammalian Sir2 homolog SIRT7 is an activator of RNA polymerase I transcription. Gene Dev. (2005) 20:1075-80. doi: 10.1101/gad.1399706

31. Kiran S, Anwar T, Kiran M, Ramakrishna G. Sirtuin 7 in cell proliferation, stress and disease: rise of the Seventh Sirtuin!. Cell Signal. (2015) 27:673-82. doi: 10.1016/j.cellsig.2014.11.026

32. Wang W, Zhang X, Zheng K, Pei Y. Sirtuin 7 plays an oncogenic role in human osteosarcoma via downregulating CDC4 expression. Am J Cancer Res. (2017) 7:1788-803.

33. Li W, Sun Z, Chen C, Wang L, Geng Z, Tao J. Sirtuin7 has an oncogenic potential via promoting the growth of cholangiocarcinoma cells. Biomed Pharmacother. (2018) 100:257-66. doi: 10.1016/j.biopha.2018.02.007

34. Gong J, Wang H, Lou W, Wang G, Tao H, Wen H, et al. Associations of sirtuins with clinicopathological parameters and prognosis in non-small cell lung cancer. Cancer Manag Res. (2018) 10:3341-56. doi: 10.2147/CMAR.S166946

35. Armour SM, Bennett EJ, Braun CR, Zhang X, McMahon SB, Gygi SP, et al. A high-confidence interaction map identifies SIRT1 as a mediator of acetylation of USP22 and the SAGA coactivator complex. Mol Cell Biol. (2012) 33:1487502. doi: 10.1128/MCB.00971-12

36. Blank MF, Grummt I. The seven faces of SIRT7. Transcription (2017) 8:67-74. doi: 10.1080/21541264.2016.1276658

37. Liu GF, Lu JY, Zhang YJ, Zhang LX, Lu GD, Xie ZJ, et al. C/EBPalpha negatively regulates SIRT7 expression via recruiting HDAC3 to the upstream-promoter of hepatocellular carcinoma cells. Biochim Biophys Acta (2016) 1859:348-54. doi: 10.1016/j.bbagrm.2015.12.004

38. Shin J, He M, Liu Y, Paredes S, Villanova L, Brown K, et al. SIRT7 represses Myc activity to suppress ER stress and prevent fatty liver disease. Cell Rep. (2013) 5:654-65. doi: 10.1016/j.celrep.2013.10.007

39. Cioffi M, Vallespinos-Serrano M, Trabulo SM, Fernandez-Marcos PJ, Firment AN, Vazquez BN, et al. MiR-93 controls adiposity via inhibition of SIRT7 and Tbx3. Cell Rep. (2015) 12:1594-605. doi: 10.1016/j.celrep.2015.08.006
40. Han Y, Liu Y, Zhang H, Wang T, Diao R, Jiang Z, et al. Hsa-miR-125b suppresses bladder cancer development by down-regulating oncogene SIRT7 and oncogenic long non-coding RNA MALAT1. FEBS Lett. (2013) 587:387582. doi: 10.1016/j.febslet.2013.10.023

41. Shi H, Ji Y, Zhang D, Liu Y, Fang P. MicroRNA-3666-induced suppression of SIRT7 inhibits the growth of non-small cell lung cancer cells. Oncol Rep. (2016) 36:3051-7. doi: 10.3892/or.2016.5063

42. Yu W, Cui X, Wan Z, Yu Y, Liu X, Jin L. Silencing forkhead box M1 promotes apoptosis and autophagy through SIRT7/mTOR/IGF2 pathway in gastric cancer cells. J Cell Biochem. (2018) 119:9090-8. doi: 10.1002/jcb.27168

43. Grob A, Roussel P, Wright JE, McStay B, Hernandez-Verdun D, Sirri V. Involvement of SIRT7 in resumption of rDNA transcription at the exit from mitosis. J Cell Sci. (2009) 122:489-98. doi: 10.1242/jcs.042382

44. Sun L, Fan G, Shan P, Qiu X, Dong S, Liao L, et al. Regulation of energy homeostasis by the ubiquitin-independent REG $\gamma$ proteasome. Nat Commun. (2016) 7:12497. doi: 10.1038/ncomms12497

45. Jiang L, Xiong J, Zhan J, Yuan F, Tang M, Zhang C, et al. Ubiquitin-specific peptidase 7 (USP7)-mediated deubiquitination of the histone deacetylase SIRT7 regulates gluconeogenesis. J Biol Chem. (2017) 292:13296-311. doi: 10.1074/jbc.M117.780130

46. Mohrin M, Shin J, Liu Y, Brown K, Luo H, Xi Y, et al. Stem cell aging. A mitochondrial UPR-mediated metabolic checkpoint regulates hematopoietic stem cell aging. Science (2015) 347:1374-7. doi: 10.1126/science.aaa2361

47. Xu C, Bailly-Maitre B, Reed JC. Endoplasmic reticulum stress: cell life and death decisions. J Clin Invest. (2005) 115:2656-64. doi: 10.1172/JCI26373

48. Zhao Q, Wang J, Levichkin IV, Stasinopoulos S, Ryan MT, Hoogenraad NJ. A mitochondrial specific stress response in mammalian cells. EMBO J. (2002) 21:4411-9. doi: 10.1093/emboj/cdf445

49. Dioum EM, Chen R, Alexander MS, Zhang Q, Hogg RT, Gerard RD, et al. Regulation of hypoxia-inducible factor $2 \alpha$ signaling by the stress-responsive deacetylase sirtuin 1. Science (2009) 324:1289. doi: 10.1126/science.1169956

50. Hubbi ME, Hu H, Kshitiz Gilkes DM, Semenza GL. Sirtuin-7 inhibits the activity of hypoxia-inducible factors. J Biol Chem. (2013) 288:20768-75. doi: 10.1074/jbc.M113.476903

51. Lewinska A, Wnuk M, Grabowska W, Zabek T, Semik E, Sikora E, et al. Curcumin induces oxidation-dependent cell cycle arrest mediated by SIRT7 inhibition of rDNA transcription in human aortic smooth muscle cells. Toxicol Lett. (2015) 233:227-38. doi: 10.1016/j.toxlet.2015.01.019

52. Araki S, Izumiya Y, Rokutanda T, Ianni A, Hanatani S, Kimura Y, et al. SIRT7 contributes to myocardial tissue repair by maintaining transforming growth factor- $\beta$ signaling pathway. Circulation (2015) 132:1081. doi: 10.1161/CIRCULATIONAHA.114.014821

53. Weber CE, Li NY, Wai PY, Kuo PC. Epithelial-mesenchymal transition, TGF$\beta$, and osteopontin in wound healing and tissue remodeling after injury. J Burn Care Res. (2012) 33:311-8. doi: 10.1097/BCR.0b013e318240541e

54. Bosch-Presegue L, Vaquero A. Sirtuins in stress response: guardians of the genome. Oncogene (2014) 33:3764-75. doi: 10.1038/onc.201 3.344

55. Vazquez BN, Thackray JK, Simonet NG, Kane Goldsmith N, Martinez Redondo P, Nguyen T, et al. SIRT7 promotes genome integrity and modulates non-homologous end joining DNA repair. EMBO J. (2016) 35:1488-503. doi: 10.15252/embj.201593499

56. Zhang P, Li G, Deng Z, Liu L, Chen L, Tang J, et al. Dicer interacts with SIRT7 and regulates $\mathrm{H} 3 \mathrm{~K} 18$ deacetylation in response to DNA damaging agents. Nucleic Acids Res. (2016) 44:3629-42. doi: 10.1093/nar/gkv1504

57. Mao Z, Hine C, Tian X, Van Meter M, Au M, Vaidya A, et al. SIRT6 promotes DNA repair under stress by activating PARP1. Science (2011) 332:1443-6. doi: 10.1126/science. 1202723

58. Hanada K, Song CZ, Yamamoto K, Yano K, Maeda Y, Yamaguchi K, et al. RNA polymerase I associated factor 53 binds to the nucleolar transcription factor UBF and functions in specific rDNA transcription. EMBO J. (1996) 15:2217-26. doi: 10.1002/j.1460-2075.1996.tb00575.x

59. Kim J, Tchernyshyov I, Semenza GL, Dang CV. HIF-1-mediated expression of pyruvate dehydrogenase kinase: a metabolic switch required for cellular adaptation to hypoxia. Cell Metab. (2006) 3:177-85. doi: 10.1016/j.cmet.2006.02.002

60. Tang BL. SIRT7 and hepatic lipid metabolism. Front Cell Dev Biol. 3:1. doi: $10.3389 /$ fcell.2015.00001 
61. Yoshizawa T, Karim MF, Sato Y, Senokuchi T, Miyata K, Fukuda T, et al. SIRT7 controls hepatic lipid metabolism by regulating the ubiquitin-proteasome pathway. Cell Metab. (2014) 19:712-21. doi: 10.1016/j.cmet.2014.03.006

62. Picard F, Kurtev M, Chung N, Topark-Ngarm A, Senawong T, Machado De Oliveira R, et al. Sirt1 promotes fat mobilization in white adipocytes by repressing PPAR-gamma. Nature (2004) 429:771-6. doi: 10.1038/nature 02583

63. Fang J, Ianni A, Smolka C, Vakhrusheva O, Nolte H, Krüger M, et al. SIRT7 promotes adipogenesis in the mouse by inhibiting autocatalytic activation of Sirt1. Proc Natl Acad Sci USA. (2017) 114:E8352-61. doi: 10.1073/pnas.1706945114

64. Poyton RO, McEwen JE. Crosstalk between nuclear and mitochondrial genomes. Annu Rev Biochem. (1996) 65:563-607. doi: 10.1146/annurev.bi.65.070196.003023

65. Vakhrusheva O, Braeuer D, Liu Z, Braun T, Bober E. SIRT7-dependent inhibition of cell growth and proliferation might be instrumental to mediate tissue integrity during aging. J Physiol Pharmacol. (2008) 59(Suppl. 9), 201-12.

66. Wronska A, Lawniczak A, Wierzbicki PM, Kmiec Z. Age-related changes in sirtuin 7 expression in calorie-restricted and refed rats. Gerontology (2016) 62:304-10. doi: 10.1159/000441603

67. Ghiraldini FG, Crispim ACV, Mello MLS. Effects of hyperglycemia and aging on nuclear sirtuins and DNA damage of mouse hepatocytes. Mol Biol Cell (2013) 24:2467-76. doi: 10.1091/mbc.e13-04-0186

68. Mortuza R, Chen S, Feng B, Sen S, Chakrabarti S. High glucose induced alteration of SIRTs in endothelial cells causes rapid aging in a p300 and FOXO regulated pathway. PLoS ONE (2013) 8:e54514. doi: 10.1371/journal.pone.0054514

69. Kiran S, Oddi V, Ramakrishna G. Sirtuin 7 promotes cellular survival following genomic stress by attenuation of DNA damage, SAPK activation and p53 response. Exp Cell Res. (2014) 331:123-41. doi: 10.1016/j.yexcr.2014.11.001

70. Colombo E, Marine J, Danovi D, Falini B, Pelicci PG. Nucleophosmin regulates the stability and transcriptional activity of p53. Nat Cell Biol. (2002) 4:529. doi: $10.1038 /$ ncb814

71. Bojesen SE. Telomeres and human health. J Intern Med. (2013) 274:399-413. doi: $10.1111 /$ joim. 12083

72. Conomos D, Stutz MD, Hills M, Neumann AA, Bryan TM, Reddel $\mathrm{RR}$, et al. Variant repeats re interspersed throughout the telomeres and recruit nuclear receptors in ALT cells. J Cell Biol. (2012) 199:893-906. doi: $10.1083 /$ jcb.201207189

73. Lu CT, Hsu CM, Lin PM, Lai CC, Lin HC, Yang CH, et al. The potential of SIRT6 and SIRT7 as circulating markers for head and neck squamous cell carcinoma. Anticancer Res. (2014) 34:7137-43.

74. Paredes S, Angulo-Ibanez M, Tasselli L, Carlson SM, Zheng W, Li TM, et al. The epigenetic regulator SIRT7 guards against mammalian cellular senescence induced by ribosomal DNA instability. J Biol Chem. (2018) 293:11242-50. doi: 10.1074/jbc.AC118.003325

75. Geng Q, Peng H, Chen F, Luo R, Li R. High expression of SIRT7 served as a predictor of adverse outcome in breast cancer. Int J Clin Exp Pathol. (2015) 8:1938-45.

76. Aljada A, Saleh AM, Alkathiri M, Shamsa HB, Al-Bawab A, Nasr A. Altered sirtuin 7 expression is associated with early stage breast cancer. Breast Cancer (Auckl). (2015) 9:3-8. doi: 10.4137/BCBCR.S23156

77. Ashraf N, Zino S, MacIntyre A, Kingsmore D, Payne AP, George WD, et al. Altered sirtuin expression is associated with node-positive breast cancer. Brit J Cancer (2006) 95:1056-61. doi: 10.1038/sj.bjc.6603384

78. Malik S, Villanova L, Tanaka S, Aonuma M, Roy N, Berber E, et al. SIRT7 inactivation reverses metastatic phenotypes in epithelial and mesenchymal tumors. Sci Rep. (2015) 5:9841. doi: 10.1038/srep09841

79. Singh S, Kumar PU, Thakur S, Kiran S, Sen B, Sharma S, et al. Expression/localization patterns of sirtuins (SIRT1, SIRT2, and SIRT7) during progression of cervical cancer and effects of sirtuin inhibitors on growth of cervical cancer cells. Tumor Biol. (2015) 36:6159-71. doi: 10.1007/s13277-015-3300-y

80. Wang HL, Lu RQ, Xie SH, Zheng H, Wen XM, Gao X, et al. SIRT7 exhibits oncogenic potential in human ovarian cancer cells. Asian Pac J Cancer Prev. (2015) 16:3573-7. doi: 10.7314/APJCP.2015.16.8.3573

81. Yu H, Ye W, Wu J, Meng X, Liu RY, Ying X, et al. Overexpression of SIRT7 exhibits oncogenic property and serves as a prognostic factor in colorectal cancer. Clin Cancer Res. (2014) 20:3434-45. doi: 10.1158/1078-0432.CCR-13-2952

82. Zhang S, Chen $\mathrm{P}, \mathrm{Huang} \mathrm{Z}, \mathrm{Hu} \mathrm{X}$, Chen $\mathrm{M}, \mathrm{Hu}$ S, et al. SIRT7 promotes gastric cancer growth and inhibits apoptosis by epigenetically inhibiting miR-34a. Sci Rep. (2015) 5:9787. doi: 10.1038/srep09787

83. McGlynn LM, McCluney S, Jamieson NB, Thomson J, MacDonald AI, Oien K, et al. SIRT3 \& SIRT7: potential novel biomarkers for determining outcome in pancreatic cancer patients. PLOS ONE (2015) 10:e131344. doi: 10.1371/journal.pone.0131344

84. Horwitz GA, Zhang K, McBrian MA, Grunstein M, Kurdistani SK, Berk AJ. Adenovirus small ela alters global patterns of histone modification. Science (2008) 321:1084-5. doi: 10.1126/science.11 55544

85. Day BW, Stringer BW, Spanevello MD, Charmsaz S, Jamieson PR, Ensbey $\mathrm{KS}$, et al. ELK4 neutralization sensitizes glioblastoma to apoptosis through downregulation of the anti-apoptotic protein Mcl-1. Neuro Oncol. (2011) 13:1202-12. doi: 10.1093/neuonc/nor119

86. Makkonen H, Jääskeläinen T, Pitkänen-Arsiola T, Rytinki M, Waltering KK, Mättö M, et al. Identification of ETS-like transcription factor 4 as a novel androgen receptor target in prostate cancer cells. Oncogene (2008) 27:4865. doi: 10.1038/onc.2008.125

87. Alvero $\mathrm{AB}$. Recent insights into the role of NF-kappaB in ovarian carcinogenesis. Genome Med. (2010) 2:56. doi: 10.1186/gm177

88. Byles V, Zhu L, Lovaas JD, Chmilewski LK, Wang J, Faller DV, et al. SIRT1 induces EMT by cooperating with EMT transcription factors and enhances prostate cancer cell migration and metastasis. Oncogene (2012) 31:4619-29. doi: 10.1038/onc.2011.612

89. Gilkes DM, Xiang L, Lee SJ, Chaturvedi P, Hubbi ME, Wirtz D, et al. Hypoxia-inducible factors mediate coordinated RhoA-ROCK1 expression and signaling in breast cancer cells. Proc Natl Acad Sci USA. (2013) 111:E38493. doi: 10.1073/pnas.1321510111

90. Paredes S, Villanova L, Chua KF. Molecular pathways: emerging roles of mammalian sirtuin SIRT7 in cancer. Clin Cancer Res. (2014) 20:1741-6. doi: 10.1158/1078-0432.CCR-13-1547

91. Haider R, Massa F, Kaminski L, Clavel S, Djabari Z, Robert G, et al. Sirtuin 7: a new marker of aggressiveness in prostate cancer. Oncotarget (2017) 8:77309-16. doi: 10.18632/oncotarget.20468

92. Tang M, Lu X, Zhang C, Du C, Cao L, Hou T, et al. Downregulation of SIRT7 by 5-fluorouracil induces radiosensitivity in human colorectal cancer. Theranostics (2017) 7:1346-59. doi: 10.7150/thno.18804

93. Burg N, Bittner S, Ellwardt E. Role of the epigenetic factor Sirt7 in neuroinflammation and neurogenesis. Neurosci Res. (2018) 131:1-9. doi: 10.1016/j.neures.2017.09.005

Conflict of Interest Statement: The authors declare that the research was conducted in the absence of any commercial or financial relationships that could be construed as a potential conflict of interest.

Copyright (c) $2018 \mathrm{Wu}, \mathrm{Li}, \mathrm{Zhu}$, Wang and Zhu. This is an open-access article distributed under the terms of the Creative Commons Attribution License (CC BY). The use, distribution or reproduction in other forums is permitted, provided the original author(s) and the copyright owner(s) are credited and that the original publication in this journal is cited, in accordance with accepted academic practice. No use, distribution or reproduction is permitted which does not comply with these terms. 